\title{
The Occurrence of Interfaces in Nonlinear Diffusion-Advection Processes
}

\author{
B. H. GILDING \\ Communicated by M. E. GURTIN
}

\section{Introduction}

The nonlinear diffusion-advection processes referred to in the title of this paper are those described by the equation

$$
u_{t}=(a(u))_{x x}+(b(u))_{x},
$$

in which subscripts denote partial differentiation. The functions $a$ and $b$ belong to $C([0, \infty)) \cap C^{2}(0, \infty)$, and are such that $a^{\prime \prime}$ and $b^{\prime \prime}$ are locally Hölder continuous on $(0, \infty)$, and $a^{\prime}(s)>0$ for $s>0$. Furthermore, without any loss of generality, it is supposed that $a(0)=0$ and $b(0)=0$. Because of its resemblance to the celebrated equation arising in statistical mechanics [9], (1.1) is often referred to as the nonlinear Fokker-Planck equation.

Equation (1.1) models a number of different physical phenomena. For instance, when $u$ denotes unsaturated soil-moisture content, the equation describes the infiltration of water in a homogeneous porous medium [5]. It also appears with $a(s)=s^{4}$ and $b(s)=-s^{3}$ in the theory of the flow of a thin viscous film over an inclined bed [7].

Equation (1.1) is parabolic when $u>0$, but may degenerate for $u=0$. Hence the equation need not admit classical solutions. Under appropriate conditions though, the equation is known to possess a unique generalized solution which is nonnegative and continuous. This solution is a classical solution of (1.1) in a neighbourhood of any point where it is positive. It is trivially also a classical solution in the interior of the set of points where it is zero $[16,22]$. However, the derivatives of the solution may be undefined or discontinuous at points separating a region where the solution is positive from one where it is zero [18, 22].

The characteristic that equation (1.1) admit solutions possessing interfaces separating a region where the solution is positive from one where it is zero, is, itself, a peculiarity associated with the degeneracy of the equation. For instance, solutions of the linear heat equation do not display such behaviour. Given an initialboundary value problem for the linear heat equation with nontrivial nonnegative initial data, the solution is positive everywhere in the problem domain [22-24]. 
In this context, the linear heat equation is often said to propagate perturbations with infinite speed. An equation which does admit solutions possessing interfaces of the type described is said to have finite speed of propagation of perturbations $[20,24]$.

In this paper we shall establish necessary and sufficient conditions for equation (1.1) to admit solutions possessing interfaces separating a region where the solution is positive from one where it is zero. We also study some properties characterizing such an interface.

For convenience, we restrict the discussion to the Cauchy problem for equation (1.1) and to interfaces which provide an upper bound for the support of a solution. Nonetheless, our results may be extended to the Cauchy-Dirichlet problem and the first boundary-value problem for equation (1.1), and to general interfaces.

To be explicit, we consider the following questions. Let $u(x, t)$ denote the continuous, nonnegative, generalized solution of the problem

$$
\begin{gathered}
u_{t}=(a(u))_{x x}+(b(u))_{x} \quad \text { for }(x, t) \in \mathbb{R} \times \mathbb{R}^{+}, \\
u(x, 0)=u_{0}(x) \quad \text { for } x \in \mathbb{R},
\end{gathered}
$$

where $u_{0}$ is a given, bounded, continuous, nonnegative function on $\mathbb{R}$. Suppose that $\sup \left\{x \in \mathbb{R}: u_{0}(x)>0\right\} \in(-\infty, \infty)$. Then, when does $\sup \{x \in \mathbb{R}: u(x, t)>0\}$ $\in(-\infty, \infty)$ for $t>0$ ? Under conditions for which this question can be answered in the affirmative, what can be stated about the interface

$$
\zeta(t)=\sup \{x \in \mathbb{R}: u(x, t)>0\}
$$

as a function of $t \geqq 0$ ? The technique we use to answer these questions is to compare $u$ with a suitably-constructed "travelling-wave solution" of equation(1.1).

The existence of the interface or free boundary $\zeta(t)$ is of substantial interest with respect to the physical situations in which equation (1.1) arises. In the instance of soil-moisture infiltration, such an interface denotes a wetting front separating wet and dry regions of the porous medium. For the flow of a thin viscous film, in which the unknown variable describes the thickness of the film, $\zeta(t)$ denotes a leading edge of the fluid flow.

Let

$$
M=\sup \left\{u_{0}(x): x \in \mathbb{R}\right\} .
$$

When equation (1.1) is replaced by the equation

$$
u_{t}=(a(u))_{x x},
$$

the interface $\zeta(t)$ exists if and only if

$$
a^{\prime}(s) / s \in L^{1}(0, M)
$$

$[23,24]$. For equation (1.1) itself, under the hypotheses

$$
a, b \in C^{1}([0, \infty)) \text {, }
$$


and $s a^{\prime \prime}(s), s b^{\prime \prime}(s) \in L^{1}(0, M),(1.5)$ is again a necessary and sufficient condition for the existence of $\zeta(t)$ [15]. However, by studying the explicit example

$$
u_{t}=\left(u^{m}\right)_{x x}+\lambda\left(u^{n}\right)_{x},
$$

with $m \geqq 1>n>0$ and $\lambda \neq 0$, DIAZ \& KERSNER [10] have recently shown that if (1.6) does not hold, the picture is not so simple. In this case, whether or not (1.5) holds (i.e. $m>1$ or $m=1$ ), the interface $\zeta(t)$ exists if and only if $\lambda>0$.

In Section 3 of this paper we shall unify the above-mentioned results by proving that the interface $\zeta(t)$ exists if and only if there is a real number $\sigma$ such that

and

$$
\sigma s+b(s)>0 \quad \text { for all } s \in(0, M]
$$

$$
a^{\prime}(s) /\{\sigma s+b(s)\} \in L^{1}(0, M) .
$$

Applied to equation (1.7) in the cases not covered in $[10,15,23,24]$, viz. $0<m$ $<1, n>0$, and $\lambda \neq 0$, this conclusion yields existence of the interface $\zeta(t)$ if and only if $n<m$ and $\lambda>0$.

In Section 5 we establish the "quasi-monotonicity" of the interface $\zeta(t)$. This generalizes its known monotonicity when equation (1.1) is restricted to the celebrated porous media equation

$$
u_{t}=\left(u^{m}\right)_{x x}
$$

or to equation (1.7) with $n>1$ or $\lambda<0$. To be specific, we shall prove that for any $t_{1} \in[0, \infty)$ and $t_{2} \in\left(t_{1}, \infty\right)$,

where

$$
\zeta\left(t_{2}\right) \geqq \zeta\left(t_{1}\right)+\sigma_{0}\left(t_{2}-t_{1}\right)
$$

$$
\sigma_{0}=\lim _{s \downarrow 0} \sup \{-b(s) / s\} .
$$

Note that $\sigma_{0}<\infty$ under the conditions for the existence of the interface $\zeta(t)$.

In Section 6, we investigate the continuity of the interface $\zeta(t)$.

Next, in Section 7, we show that the interface $\zeta(t)$ satisfies the equation

$$
\zeta^{\prime}(t)=-\left\{\left[(a(u))_{x}+b(u)\right] / u\right\}(\zeta(t)-, t)
$$

in a certain sense. This identity is to be expected with regard to the physical situations in which equation (1.1) arises. In the setting of soil-moisture infiltration, the condition states that the wetting front $\zeta(t)$ moves at a speed equal to the particle velocity of the moisture approaching it. Likewise, for the flow of a thin viscous film over an inclined bed, the condition states that the leading edge moves at a speed equal to that of the fluid particles converging upon it.

Finally, in Section 8 , we strengthen the results on the continuity of the interface $\zeta(t)$ established in Section 6. In particular, under the assumption that (1.5) holds, we obtain Hölder and Lipschitz continuity estimates for the function $\zeta(t)$. These generalize estimates previously obtained for interfaces of equation (1.7) with $m>1$ and $n \geqq(m+1) / 2$ in [14].

Before we can prove any of the above results we need to complete some groundwork. In the next section we clarify the idea of a solution of equation (1.1) 
and summarize the theory we need concerning existence uniqueness, regularity and comparison principles for solutions of the equation. We also prove two preliminary propositions. The second of these is a regularity estimate for solutions of equation (1.1) which is sharper than earlier results of this kind.

We remark that necessary and sufficient conditions for finite speed of propagation of perturbations for the equation

$$
u_{t}=(a(u))_{x x}+c(u)
$$

with $c(u)$ negative have been established in [20]. Furthermore, for the semilinear multidimensional equivalent of (1.9) and for the equation

$$
u_{t}=(a(u))_{x x}+(b(u))_{x}+c(u)
$$

where $a$ and $b$ satisfy (1.6) and $c$ is again negative, sufficient conditions for the existence of the corresponding interfaces and some of their properties have been investigated in [13]. In addition, sufficient conditions for the finite speed of propagation of perturbations for a very broad class of quasilinear parabolic equations in an arbitrary number of spatial dimensions have been established in [2, 11, 12].

We also remark that for the porous media equation (1.8), a great deal more than is suggested by the results of the present paper is known about the behaviour of the interface $\zeta(t)$. For instance, it is known that there is a waiting time $t^{*} \in[0, \infty)$ such that $\zeta(t)$ is constant on $\left[0, t^{*}\right]$ and strictly monotonic increasing on $\left[t^{*}, \infty\right)$. Moreover $\zeta \in C^{\infty}\left((0, \infty) \backslash\left\{t^{*}\right\}\right)$ and

$$
D^{+} \zeta(t)=\frac{-m}{m-1}\left(u^{m-1}\right)_{x}(\zeta(t)-, t)
$$

for all $t>0$. Further details can be found in $[1,3,4,6,8,17,21,26,27]$.

\section{Preliminaries}

Let $D$ denote the domain

with

$$
D=\left(\eta_{1}, \eta_{2}\right) \times\left(\tau_{1}, \tau_{2}\right]
$$

$$
-\infty \leqq \eta_{1}<\eta_{2} \leqq \infty \quad \text { and } \quad 0 \leqq \tau_{1}<\tau_{2}<\infty
$$

Definition 1. A function $u(x, t)$ is said to be a generalized supersolution of equation (1.1) in $D$ if $u$ is defined, real, nonnegative, bounded, and continuous in $\bar{D}$, and satisfies the integral inequality

$$
\begin{aligned}
\int_{t_{1}}^{t_{2}} \int_{x_{1}}^{x_{2}}\left\{u \phi_{t}+a(u)\right. & \left.\phi_{x x}-b(u) \phi_{x}\right\} d x d t \\
\leqq & \int_{x_{1}}^{x_{2}}\left\{u\left(x, t_{2}\right) \phi\left(x, t_{2}\right)-u\left(x, t_{1}\right) \phi\left(x, t_{1}\right)\right\} d x \\
& +\int_{t_{1}}^{t_{2}}\left\{a\left(u\left(x_{2}, t\right)\right) \phi_{x}\left(x_{2}, t\right)-a\left(u\left(x_{1}, t\right)\right) \phi_{x}\left(x_{1}, t\right)\right\} d t
\end{aligned}
$$


for all nonempty bounded rectangles $R=\left(x_{1}, x_{2}\right) \times\left(t_{1}, t_{2}\right] \leqq D$ and nonnegative functions $\phi \in C^{2,1}(\bar{R})$ such that $\phi\left(x_{1}, t\right)=\phi\left(x_{2}, t\right)=0$ for all $t \in\left[t_{1}, t_{2}\right]$. Similarly, a function $u(x, t)$ is said to be a generalized subsolution of equation (1.1) in $D$ if it meets the above requirements with the inequality (2.3) reversed. A function is a generalized solution of equation (1.1) in $D$ if it is a generalized supersolution and subsolution.

Definition 2. A function $u(x, t)$ is a generalized solution of problem (1.2), (1.3) if it satisfies (1.3) and is a generalized solution of equation (1.1) in $(-\infty, \infty) \times$ $(0, T]$ for any $T>0$.

Lemma 1 (Existence and uniqueness) [16]. Suppose that $a, b \in C([0, \infty)) \cap$ $C^{2}(0, \infty)$,

$$
\begin{aligned}
& a(0)=0, \quad b(0)=0, \\
& a^{\prime}(s)>0 \quad \text { for } s>0,
\end{aligned}
$$

and, $a^{\prime \prime}$ and $b^{\prime \prime}$ are locally Hölder continuous on $(0, \infty)$, and that $u_{0}$ is bounded, nonnegative, and continuous on $\mathbb{R}$. Then problem (1.2), (1.3) admits a unique generalized solution.

To avoid unnecessary repetition, THROUGHOUT THE REMAINDER OF THIS PAPER, IT WILL AUTOMATICALLY BE ASSUMED THAT THE HYPOTHESES OF LEMMA 1 ARE SATISFIED. The letter $H$ will denote the half-space

$$
H=\mathbb{R} \times \mathbb{R}^{+},
$$

$M$ will denote the supremum of the initial data function $u_{0}$ as defined in (1.4), and $u(x, t)$ will denote the unique generalized solution of problem (1.2), (1.3). Furthermore, for any variable $y$, we let

$$
[y]_{+}=\max \{0, y\} \text {. }
$$

Lemma 2 (Regularity) [16]. In the sense of distributions, $(a(u))_{x}$ exists and is bounded on any set of the form $(-\infty, \infty) \times[\tau, \infty), \tau>0$. In particular, if $a\left(u_{0}\right)$ is uniformly Lipschitz continuous on $\mathbb{R}$, then, in the sense of distributions, $(a(u))_{x}$ exists and is bounded in $\bar{H}=(-\infty, \infty) \times[0, \infty)$. Furthermore, $u$ and $(a(u))_{x} \in C^{2,1}(P)$, where $P=\{(x, t) \in H: u(x, t)>0\}$, and $u$ is a classical solution of equation (1.1) in $P$.

Lemma 3 (Comparison principle) [16]. Let $D$ be a domain of the form (2.1), (2.2). (a) If $U(x, t)$ denotes a generalized supersolution of equation (1.1) in $D$ such that $U(x, t)>0$ for all $(x, t) \in \bar{D} \backslash\left(\eta_{1}, \eta_{2}\right) \times\left[\tau_{1}, \tau_{2}\right]$ and $U(x, t) \geqq u(x, t)$ for all $(x, t) \in \bar{D} \backslash D$, then $U(x, t) \geqq u(x, t)$ for all $(x, t) \in \bar{D}$.

(b) If $U(x, t)$ denotes a generalized subsolution of equation (1.1) in $D$ such that $U(x, t) \leqq u(x, t)$ for all $(x, t) \in \bar{D} \backslash D$, then $U(x, t) \leqq u(x, t)$ for all $(x, t) \in \bar{D}$. 
Corollary.

$$
u(x, t) \leqq M \quad \text { for all }(x, t) \in \bar{H}
$$

As a useful alternative to Lemma 3, we also have the following.

Lemma 4. Let $\Omega$ denote $a$ domain of the form $\Omega=\left\{(x, t): \xi_{1}(t)<x<\xi_{2}(t)\right.$, $\left.\tau_{1}<t \leqq \tau_{2}\right\}$ where $0 \leqq \tau_{1}<\tau_{2}<\infty$, and $\xi_{1}$ and $\xi_{2}$ are $C\left(\left[\tau_{1}, \tau_{2}\right]\right)$ functions such that $\xi_{1}(t)<\xi_{2}(t)$ for all $t \in\left(\tau_{1}, \tau_{2}\right]$. Then if $U \in C(\bar{\Omega})$ denotes a positive classical subsolution of equation (1.1) in $\Omega$ such that $U(x, t) \leqq u(x, t)$ for all $(x, t) \in \bar{\Omega} \backslash \Omega, \quad U(x, t) \leqq u(x, t)$ for all $(x, t) \in \bar{\Omega}$.

Lemma 4 follows from the constructive method of proving existence in [16].

The next result is of some inherent interest. In the light of the physical applications of equation (1.1), it can be interpreted as the verification of conservation of mass in nonlinear diffusion-advection processes; $c f$. [14].

Proposition 1. For any $t>0$

$$
\int_{-\infty}^{\infty} u(x, t) d x=\int_{-\infty}^{\infty} u_{0}(x) d x
$$

Proof. We adapt an earlier proof of the proposition for equation (1.7) with $m>1$ and $n \geqq(m+1) / 2$ in [14].

Fix $t>0$. For $i \geqq 1$, define the function $\phi_{i} \in C^{2}([-i, i])$ by

$$
\phi_{i}(x)= \begin{cases}1 & \text { if }|x| \leqq i-1 \\ (i-|x|)^{2}\left\{3(|x|-i+1)^{2}+2(|x|-i+1)+1\right\} & \text { if }|x|>i-1\end{cases}
$$

and set $R_{i}=(-i, i) \times(0, t]$. Substituting the test-function $\phi_{i}$ defined on the rectangle $R_{i}$ into the definition of a generalized solution of problem (1.2), (1.3) yields

$$
\begin{aligned}
\mid \int_{-i}^{i} \phi_{i}(x)\{u(x, t) & \left.-u_{0}(x)\right\} d x \mid \\
& =\left|\int_{S_{i, t}}\left\{a(u(x, s)) \phi_{i}^{\prime \prime}(x)-b(u(x, s)) \phi_{i}^{\prime}(x)\right\} d x d s\right| \\
& \leqq \iint_{S_{i, t}}\{12 a(u(x, s))+2|b(u(x, s))|\} d x d s \\
& \leqq 2 C t
\end{aligned}
$$

for all $i \geqq 1$, where

$$
S_{i, t}=\{(-i,-i+1) \cup(i-1, i)\} \times(0, t]
$$


and

$$
C=\sup \{12 a(r)+2|b(r)|: 0 \leqq r \leqq M\} .
$$

Hence, letting $i \uparrow \infty$, we see that $u(., t) \in L^{1}(\mathbb{R})$ if and only if $u_{0} \in L^{1}(\mathbb{R})$.

Suppose now that $u_{0} \in L^{1}(\mathbb{R})$. Note that for any $s>0$, there is a constant $K=K(s)>0$ such that

$$
|a(u(x, s))-a(u(y, s))| \leqq K|x-y| \text { for all } x, y \in \mathbb{R},
$$

by Lemma 2 . Hence, since $u(., s) \in L^{\mathbf{1}}(\mathbb{R}), u(x, s) \rightarrow 0$ as $|x| \rightarrow \infty$ for all $s>0$. However, in view of (2.4), this means that for every $s>0,\{12 a(u(x, s))$ $+2|b(u(x, s))|\} \rightarrow 0$ as $|x| \rightarrow \infty$. Subsequently, letting $i \uparrow \infty$ in (2.7), and applying the Dominated Convergence Theorem, we see that (2.6) must hold.

Proposition 2. (i) Suppose that $[-b(s) / s]_{+} \in L^{\infty}(0, M)$ and $a^{\prime}(s) / s \in L^{1}(0, M)$. Then, given any $\tau>0$, there is a constant $C>0$, which depends only on $M$ and $\tau$, such that

$$
-\left\{(a(u))_{x}+b(u)\right\} \leqq C u
$$

in $(-\infty, \infty) \times[\tau, \infty)$, in the sense of distributions.

(ii) Suppose that $[-b(s) / s]_{+} \in L^{\infty}(0, M)$, and $a^{\prime}(s) /\{L s+b(s)\} \in L^{1}(0, M)$ for some $L>\sup \{-b(s) / s: 0<s \leqq M\}$. Suppose furthermore that

$$
\int_{u_{0}\left(x_{2}\right)}^{u_{0}\left(x_{1}\right)} a^{\prime}(s) /\{L s+b(s)\} d s \leqq x_{2}-x_{1}
$$

for all $x_{1}, x_{2} \in \mathbb{R}$ with $x_{2}>x_{1}$. Then there is a constant $C>0$ which depends only on $M$ and $L$ such that $(2.8)$ holds in $\bar{H}=(-\infty, \infty) \times[0, \infty)$ in the sense of distributions.

Proof. If $\tilde{M} \in(M, \infty)$, then [16] $u(x, t)$ can be constructed on any set of the form $(-\infty, \infty) \times[0, T], T \in(0, \infty)$, as the pointwise limit of a sequence of functions, $\left\{u_{k}\right\}_{k=k_{0}}^{\infty}$, with the following properties:

(i) $u_{k}$ is defined on the closure of the set

$$
S_{k}=(-k, k) \times(0, T] ;
$$

(ii) $u_{k} \in C^{2,1}\left(\bar{S}_{k}\right),\left(a\left(u_{k}\right)\right)_{x} \in C^{2,1}\left(S_{k}\right)$ and $u_{k}$ is a classical solution of equation (1.1) in $\bar{S}_{k}$;

(iii) there is an $\varepsilon_{k}>0$ such that $\varepsilon_{k} \leqq u_{k}(x, t) \leqq \tilde{M}$ for all $(x, t) \in \bar{S}_{k}$;

(iv) $u_{k}(x, 0)=\tilde{M}$ for $k-1 / 2 \leqq|x| \leqq k$ and $u_{k}( \pm k, t)=\tilde{M}$ for $t \in[0, T]$;

(v) $u_{k} \downarrow u$ as $k \uparrow \infty$ uniformly on compact subsets of $(-\infty, \infty) \times[0, T]$;

(vii) if (2.9) holds, there is a constant $L^{*}$, which does not depend on $k$, such that

$$
v_{k}(x, 0) \leqq L^{*} \quad \text { for all } x \in[-k, k],
$$

where

$$
v_{k}(x, t)=-\left\{\left[\left(a\left(u_{k}\right)\right)_{x}+b\left(u_{k}\right)\right] / u_{k}\right\}(x, t) .
$$


Moreover [16], there is a constant $K>0$ such that

$$
\left|v_{k}( \pm k, t)\right| \leqq K \quad \text { for all } t \in[0, T] .
$$

Proposition 2 is consequently a corollary of the following lemma.

Lemma 5. (i) Suppose that (2.11) holds for some $K>0$. Then there is a constant $C>0$ which depends only on $\tilde{M}$ and $K$ such that

$$
v_{k}(x, t) \leqq C \max \left\{t^{-1 / 2}, 1\right\} \quad \text { for all }(x, t) \in S_{k} .
$$

(ii) Suppose that (2.10) and (2.11) hold for some $L^{*}>0$ and $K>0$. Then there is a constant $C>0$ which depends only on $L^{*}$ and $K$ such that

$$
v_{k}(x, t) \leqq C \quad \text { for all }(x, t) \in \bar{S}_{k} .
$$

Proof. For convenience we drop the subscript $k$. We use an adaptation of the Bernstein technique proposed by BÉNILAN, $c f$. [16]. Set

$$
p(x, t)=-\left\{\left[(a(u))_{x}+b(u)\right] / \theta(u)\right\}(x, t)
$$

where $\theta$ is a positive, twice continuously differentiable function on $(0, \tilde{M}]$. Then [16] $p$ satisfies

$$
N(p)=0 \text { in } S,
$$

where $N$ is the nonlinear parabolic differential operator

$$
\begin{aligned}
N(p)= & a^{\prime}(u) p_{x x}-\left\{2 \theta^{\prime}(u)+a^{\prime \prime}(u) \frac{\theta(u)}{a^{\prime}(u)}\right\} p p_{x} \\
& +\left\{b^{\prime}(u)-a^{\prime \prime}(u) \frac{b(u)}{a^{\prime}(u)}-2 b(u) \frac{\theta^{\prime}(u)}{\theta(u)}\right\} p_{x} \\
& +\theta(u) \frac{\theta^{\prime \prime}(u)}{a^{\prime}(u)} p^{3}+2 b(u) \frac{\theta^{\prime \prime}(u)}{a^{\prime}(u)} p^{2}+\frac{b^{2}(u)}{a^{\prime}(u)} \frac{\theta^{\prime \prime}(u)}{\theta(u)} p-p_{t} .
\end{aligned}
$$

(i) Set

$$
\theta(s)=\int_{0}^{s}\{\Theta-\tilde{a}(r)\} d r
$$

where

$$
\begin{aligned}
& \Theta=1+\beta+\tilde{a}(\tilde{M}), \\
& \tilde{a}(s)=\int_{0}^{s} a^{\prime}(r) / r d r
\end{aligned}
$$

and

$$
\beta=\sup \left\{[-b(s) / s]_{+}: 0<s \leqq \tilde{M}\right\}
$$


Observe that since $\tilde{a}$ increases monotonically,

$$
s, \beta s \leqq O(s) \leqq \Theta s \quad \text { for all } s \in(0, \tilde{M}],
$$

and hence

$$
|p(x, t)| \leqq|v(x, t)| \leqq \Theta|p(x, t)| \quad \text { for all }(x, t) \in \bar{S}
$$

Moreover, by differentiation,

$$
\theta^{\prime}(x)=\Theta-\tilde{a}(s)
$$

and

$$
\theta^{\prime \prime}(s)=-a^{\prime}(s) / s
$$

Hence, for all $s \in(0, M]$ :

$$
\begin{gathered}
1<\theta^{\prime}(s)<\theta(s) / s, \\
\theta^{\prime \prime}(s)<0 \\
a^{\prime}(s)=-s \theta^{\prime \prime}(s) \leqq-\theta(s) \theta^{\prime \prime}(s),
\end{gathered}
$$

and

$$
-b(s) \leqq[-b(s)]_{+} \leqq \beta s \leqq \theta(s)
$$

Thus, putting

$$
z(x, t)=C_{0} f(t)
$$

where $C_{0}>0$ and

$$
f(t)= \begin{cases}4 t^{-1 / 2} & \text { if } 0<t \leqq 1 \\ 3+(2-t)^{2} & \text { if } 1<t \leqq 2 \\ 3 & \text { if } 2<t\end{cases}
$$

into (2.12), and noting that

$$
1 \leqq f(t) \quad \text { and } \quad 0 \leqq-f^{\prime}(t) \leqq f^{3}(t) \quad \text { for all } t>0,
$$

we see that

$$
\begin{aligned}
N(z)= & -\theta(u) \theta^{\prime \prime}(u)\left\{-C_{0}^{3} f^{3}-\frac{2 b(u)}{\theta(u)} C_{0}^{2} f^{2}\right. \\
& \left.-\frac{b^{2}(u)}{\theta^{2}(u)} C_{0} f+\frac{a^{\prime}(u)}{\theta(u) \theta^{\prime \prime}(u)} C_{0} f^{\prime}\right\} / a^{\prime}(u) \\
\leqq & -\theta(u) \theta^{\prime \prime}(u)\left\{-C_{0}^{3} f^{3}+2 C_{0}^{2} f^{2}-C_{0} f^{\prime}\right\} / a^{\prime}(u) \\
\leqq & -C_{0}^{3} f^{3} \theta(u) \theta^{\prime \prime}(u)\left\{-1+2 C_{0}^{-1}+C_{0}^{-2}\right\} / a^{\prime}(u)
\end{aligned}
$$

It follows that if $C_{0}$ is large enough,

$$
N(z) \leqq 0 \quad \text { in } S
$$

and

$$
z( \pm k, t) \geqq C_{0} \geqq p( \pm k, t) \quad \text { for all } t \in(0, T] \text {. }
$$


Hence, observing that $f(t) \uparrow \infty$ as $t \downarrow 0$, by the standard maximum principle for nonlinear parabolic equations [25] we conclude that

$$
z(x, t) \geqq p(x, t) \quad \text { for all }(x, t) \in S .
$$

Thence

$$
v(x, t) \leqq \Theta z(x, t) \quad \text { for all }(x, t) \in S .
$$

(ii) Take $\theta(s) \equiv s$ and $z(x, t) \equiv \max \left\{K, L^{*}\right\}$ in the preceding argument.

In the derivation of equation (1.1) in the theory of unsaturated soil-moisture flow, the variable $-\left\{\left[(a(u))_{x}+b(u)\right] / u\right\}(x, t)$ can be interpreted as the soilmoisture particle velocity.

\section{Necessary and Sufficient Conditions}

Theorem 1. Suppose that

$$
\sup \left\{x \in \mathbb{R}: u_{0}(x)>0\right\} \in(-\infty, \infty)
$$

Then for any $t>0$,

$$
\sup \{x \in \mathbb{R}: u(x, t)>0\} \in(-\infty, \infty)
$$

if and only if there is a real number $\sigma$ such that

$$
\sigma s+b(s)>0 \quad \text { for all } s \in(0, M]
$$

and

$$
a^{\prime}(s) /\{\sigma s+b(s)\} \in L^{1}(0, M)
$$

Remark. For given functions $a$ and $b$, and for arbitrary $M>0$, let

$$
S_{M}=\{\sigma \in \mathbb{R}: \text { (3.3) and (3.4) hold }\} .
$$

Then either $S_{M}$ is empty for all $M>0$, or $S_{M}$ is not empty for all $M>0$. Moreover, if $S_{M}$ is not empty, then

$$
\sigma_{M}=\sup \{-b(s) / s: 0<s \leqq M\}
$$

is finite, and

$$
\left(\sigma_{M}, \infty\right) \subseteq S_{M} \subseteq\left[\sigma_{M}, \infty\right)
$$

Either inclusion may be strict.

To confirm this remark we make a number of observations. Firstly, if $\sigma^{*} \in S_{M}$ for some $M>0$ then trivially $\left[\sigma^{*}, \infty\right) \leqq S_{M} \leqq S_{M^{\prime}}$ for any $M^{\prime} \in(0, M)$, whilst $\left[\sigma^{*}, \infty\right) \cap\left(\sigma_{M}, \infty\right) \subseteq S_{M}$, for any $M^{\prime} \in(M, \infty)$. Secondly, if $\sigma^{*} \in S_{M}$ 
for some $M>0$ then

$$
\begin{aligned}
\infty & >\int_{0}^{M} a^{\prime}(s) /\left\{\sigma^{*} s+b(s)\right\} d s \\
& =\left(\sigma^{*}-\sigma_{M}\right)^{-1} \int_{0}^{M} a^{\prime}(s) /\left\{s+\left(\sigma^{*}-\sigma_{M}\right)^{-1}\left[\sigma_{M} s+b(s)\right]\right\} d s \\
& \geqq\left(\sigma^{*}-\sigma_{M}\right)^{-1} \int_{0}^{M} a^{\prime}(s) /\left\{s+\left(\sigma-\sigma_{M}\right)^{-1}\left[\sigma_{M} s+b(s)\right]\right\} d s \\
& =\left(\sigma-\sigma_{M}\right)\left(\sigma^{*}-\sigma_{M}\right)^{-1} \int_{0}^{M} a^{\prime}(s) /\{\sigma s+b(s)\} d s
\end{aligned}
$$

for any $\sigma \in\left(\sigma_{M}, \sigma^{*}\right)$. Finally, when $a(s)=s^{m}$ and $b(s)=s^{n}(1-s)$, with $m>n>1, \quad \sigma_{M}=0 \in S_{M}$ for all $M<1$, whilst $\sigma_{M}=M^{n-1}(M-1) \notin S_{M}$ for all $M \geqq 1$.

In the light of the above remark, Theorem 1 is a simple consequence of Proposition 1 and the following two lemmas.

Lemma 6. Suppose that $S_{M}$ is not empty and that for some $\left(x_{0}, t_{0}\right) \in \bar{H}$,

$$
u\left(x, t_{0}\right)=0 \quad \text { for all } x \geqq x_{0} \text {. }
$$

Then, given any $\sigma>\sigma_{M}$ and $t>t_{0}$,

$$
u(x, t)=0 \quad \text { for all } x \geqq x_{0}+\sigma\left(t-t_{0}\right)+\int_{0}^{M} a^{\prime}(s) /\{\sigma s+b(s)\} d s .
$$

Lemma 7. Suppose that $S_{M}$ is empty. If $(x, t) \in H$ is such that $u(x, t)>0$, then $u\left(x^{\prime}, t\right)>0$ for all $x^{\prime} \geqq x$.

Proof of Lemma 6. Fix $\sigma>\sigma_{M}$ and set

$$
\delta=\int_{0}^{M} a^{\prime}(s) /\{\sigma \sigma+b(s)\} d s .
$$

Without loss of generality suppose that $\left(x_{0}, t_{0}\right)=(0,0)$. Consider the function $z(x, t)$ defined on $\bar{H}$ by

$$
\begin{gathered}
z(x, t)=M \quad \text { for } x \leqq \sigma t, \\
\int_{z(x, t)}^{M} a^{\prime}(s) /\{\sigma s+b(s)\} d s=x-\sigma t \quad \text { for } \sigma t<x<\sigma t+\delta, \\
z(x, t)=0 \quad \text { for } x \geqq \sigma t+\delta .
\end{gathered}
$$

It can be verified that $z(x, t)$ is a generalized supersolution of equation (1.1) in $(-\infty, \infty) \times(0, T]$ for any $T>0$. Indeed, $z$ is a classical travelling wave solution 
of equation (1.1) in $H \backslash\{(\sigma t, t),(\sigma t+\delta, t): t \geqq 0\}$. Moreover, $u(x, 0) \leqq M=$ $z(x, 0)$ for all $x \leqq 0$ and $u(x, 0)=0 \leqq z(x, 0)$ for all $x \geqq 0$. Hence, by Lemma $3, u(x, t) \leqq z(x, t)$ for all $(x, t) \in \bar{H}$.

Proof of Lemma 7. Suppose that the lemma is false and there are points $\left(x_{1}, t_{1}\right)$, $\left(x_{2}, t_{1}\right) \in H$ such that $x_{1}<x_{2}$,

$$
u\left(x_{1}, t_{1}\right)>0 \quad \text { and } \quad u\left(x_{2}, t_{1}\right)=0 .
$$

Let $\mu>0$ and $t_{0} \in\left[0, t_{1}\right)$ be such that

$$
u\left(x_{1}, t\right) \geqq \mu>0 \quad \text { for all } t \in\left[t_{0}, t_{1}\right] .
$$

Choose $\sigma$ such that

$$
\sigma>\left|x_{2}-x_{1}\right| /\left|t_{1}-t_{0}\right|
$$

set

$$
\beta_{0}=\sup \{-\sigma s-b(s): 0 \leqq s \leqq \mu\}
$$

and consider the integral

$$
I(\beta)=\int_{0}^{\mu} a^{\prime}(s) /\{\sigma s+b(s)+\beta\} d s
$$

for $\beta>\beta_{0}$. We assert that $I(\beta) \uparrow \infty$ as $\beta \downarrow \beta_{0}$.

Observe that $I(\beta)$ is a monotonic decreasing function of $\beta>\beta_{0}$. Hence, by the Monotone Convergence Theorem, if the aforementioned assertion is not true, $I\left(\beta_{0}\right)$ exists and is finite. However, recalling (2.4) and the definition of $\beta_{0}$, we see that either (i) there is an $s_{0} \in(0, \mu]$ such that $\beta_{0}=-\sigma s_{0}-b\left(s_{0}\right)$; or (ii) $\beta_{0}=0>-\sigma s-b(s)$ for all $s \in(0, \mu]$. In the former case it can be easily verified that the integral $I\left(\beta_{0}\right)$ is singular, since by $(2.5), a^{\prime}\left(s_{0}\right)>0$. In the latter case, if $I\left(\beta_{0}\right)=I(0)$ is finite, then $\sigma \in S_{\mu}$. This contradicts the assumption that $S_{M}$ is empty in view of the remark following the statement of Theorem 1 . Thus by reductio ad absurdum, $I(\beta) \uparrow \infty$ as $\beta \downarrow \beta_{0}$.

Select a $\beta>\beta_{0}$ such that

$$
I(\beta)>\sigma\left|t_{1}-t_{0}\right|
$$

Let $z(x, t)$ denote the function defined on $\left[x_{1}, \infty\right) \times\left[t_{0}, t_{1}\right]$ by

$$
\int_{0}^{z(x, t)} a^{\prime}(s) /\{\sigma s+b(s)+\beta\} d s=\left[\sigma\left(t-t_{0}\right)-x+x_{1}\right]_{+} .
$$

One can show that $z(x, t)$ is a classical travelling wave solution of equation (1.1) in $\left[\left(x_{1}, \infty\right) \backslash\left\{x_{1}+\sigma\left(t-t_{0}\right)\right\}\right] \times\left(t_{0}, t_{1}\right]$ and consequently a generalized subsolution of equation (1.1) in $\left(x_{1}, \infty\right) \times\left(t_{0}, t_{1}\right]$. Moreover, $z\left(x, t_{0}\right)=0 \leqq u\left(x, t_{0}\right)$ for all $x \in\left[x_{1}, \infty\right)$, and in view of $(3.6), z\left(x_{1}, t\right) \leqq \mu \leqq u\left(x_{1}, t\right)$ for all $t \in\left[t_{0}, t_{1}\right]$. 
Lemma 3 therefore implies that $u(x, t) \geqq z(x, t)$ for all $(x, t) \in\left[x_{1}, \infty\right) \times\left[t_{0}, t_{1}\right]$. However, by the particular choice of $\sigma$ in (3.5), this means that $u\left(x_{2}, t_{1}\right) \geqq$ $z\left(x_{2}, t_{1}\right)>0$.

Thus the assumption that the lemma is false leads to a contradiction.

\section{Intermezzo}

In the preceding section we have established necessary and sufficient conditions for the generalized solution $u(x, t)$ of problem (1.2), (1.3) to possess an interface defining the least upper bound of the points $x \in \mathbb{R}$ where $u(x, t)>0$. In the remainder of this paper we shall be concerned with the properties of this interface. Thus, HENCEFORTH, IT WILL BE SUPPOSED THAT (3.1) HOLDS AND THAT $S_{M}=\{\sigma \in \mathbb{R}$ : (3.3) and (3.4) hold $\}$ IS NOT EMPTY. We may therefore define the interface

$$
\zeta(t)=\sup \{x \in \mathbb{R}: u(x, t)>0\}
$$

for all $t \geqq 0$. We adopt and retain the notation

$$
\sigma_{M}=\sup \{-b(s) / s: 0<s \leqq M\}
$$

and

$$
\sigma_{0}=\limsup _{s \downarrow 0}\{-b(s) / s\}=\lim _{M \downarrow 0} \sigma_{M} .
$$

By the remark in the previous section: $-\infty<\sigma_{M}<\infty$ and

$$
S_{M}=\left[\sigma_{M}, \infty\right) \text { or } \quad S_{M}=\left(\sigma_{M}, \infty\right)
$$

for all $M>0$. Plainly $-\infty \leqq \sigma_{0} \leqq \sigma_{M}$ for any $M>0$.

\section{Quasi-Monotonicity}

Theorem 2. For any $t_{1} \in[0, \infty)$ and $t_{2} \in\left(t_{1}, \infty\right)$,

$$
\zeta\left(t_{2}\right) \geqq \zeta\left(t_{1}\right)+\sigma_{0}\left(t_{2}-t_{1}\right) .
$$

To prove this theorem we use the following two lemmas.

Lemma 8. Let $T>0$ and $v=\sup \{u(x, \tau): x \in \mathbb{R}\}>0$. Then given any $\mu \in(0, \nu)$ there is a $\xi \in C([0, T])$ such that $u(\xi(t), t)>\mu$ for all $t \in[0, T]$.

Proof. For $k \geqq 1$, set

$$
u_{0, k}(x)= \begin{cases}u_{0}(x) & \text { for }|x| \leqq k-1 \\ {[k-|x|]_{+} u_{0}(x)} & \text { for }|x|>k-1\end{cases}
$$

and let $u_{k}(x, t)$ denote the generalized solution of equation (1.1) in

$$
Q_{k}=(-k, k) \times(0, T],
$$


which satisfies

$$
\begin{array}{cc}
u_{k}(x, 0)=u_{0, k}(x) & \text { for } x \in[-k, k] \\
u_{k}( \pm k, t)=0 & \text { for } t \in(0, T]
\end{array}
$$

and $u_{k}$ is a classical solution of equation (1.1) in

$$
P_{k}=\left\{(x, t) \in Q_{k}: u_{k}(x, t)>0\right\} .
$$

whose existence is proved in [16]. Evoking arguments in [16], one can show that

$$
u_{k}(x, t) \uparrow u(x, t) \quad \text { as } k \uparrow \infty
$$

uniformly on compact subsets of $(-\infty, \infty) \times[0, T]$.

Let $X \in \mathbb{R}$ be such that $u(X, T)>\mu$, and choose $k \geqq 1$ so that $u_{k}(X, T)$ $>\mu$. Next, let $G$ denote the connected component of the set $\left\{(x, t) \in \bar{Q}_{k}\right.$ : $\left.u_{k}(x, t)>\mu\right\}$ to which the point $(X, T)$ belongs. Because $u_{k}$ is a classical solution of equation (1.1) in $P_{k}$ and satisfies (5.3), by the maximum principle for parabolic equations [25] there must be an $x_{0} \in(-k, k)$ such that $\left(x_{0}, 0\right) \in G$. However, because $G$ is open and connected in the topology of $\bar{Q}_{k}$, this means that there is a piecewise linear path

$$
C=\{(x(s), t(s)): 0 \leqq s \leqq 1\} \leqq G
$$

such that

$$
(x(0), t(0))=\left(x_{0}, 0\right) \quad \text { and } \quad(x(1), t(1))=(X, T) .
$$

We assert that the path $G$ of the form (5.4), (5.5) can be so chosen that $t(s)$ increases strictly monotonically on $[0,1]$. Suppose to begin with that the function $t(s)$ of the derived path $C$ is not monotonic on $[0,1]$. Then in view of (5.5) there are values $s_{1} \in(0,1)$ and $s_{2} \in\left(s_{1}, 1\right]$ such that $t(s)<t\left(s_{1}\right)=t\left(s_{2}\right)$ for all $s \in\left(s_{1}, s_{2}\right)$. Moreover, at least one of these values can be so chosen that it corresponds with a point where different line segments of $C$ are pieced together. Let $\Omega$ denote the largest subset of $Q_{k}$ whose parabolic boundary is $\Gamma=\{(x(s)$, $\left.t(s)): s_{1} \leqq s \leqq s_{2}\right\}$ and let $\mu^{*}=\inf \left\{u_{k}(x, t):(x, t) \in \Gamma\right\}>\mu$. Since $\mu^{*}$ is a classical solution of equation (1.1) in $\Omega$ and in [16] $u_{k}$ is constructed as the limit of a sequence of positive classical solutions of (1.1) in $Q_{k}$, applying the maximum principle to this sequence and taking limits shows that $u_{k}(x, t) \geqq \mu^{*}>\mu$ for all $(x, t) \in \bar{\Omega}$. However, this means that if one replaces $I$ by the straight line joining its end-points, this creates an alternative path which is piecewise linear and satisfies (5.4) and (5.5). Subsequently, repeating this procedure as often as necessary, one may construct a piecewise linear path that satisfies (5.4) and (5.5) and is such that $t(s)$ is monotonic on $[0,1]$. However, given that $t(s)$ is monotonic, in view of the compactness of the path, by modifying the line segments on which $t(s)$ is not strictly increasing, one may define a new path of the type (5.4), (5.5) which we again denote by $C$, such that $t(s)$ is strictly monotonic on $[0,1]$.

Given a path $C$ of the type (5.4), (5.5) with $t(s)$ strictly monotonic increasing, define $\xi \in C([0, T])$ by $\{(\xi(t), t): 0 \leqq t \leqq T\}$. By construction, $u(\xi(t), t) \geqq$ $u_{k}(\xi(t), t)>\mu$ for all $t \in[0, T]$. 
Lemma 9. If $\sigma_{0}>-\infty$ and $P_{0}=\left\{x \in \mathbb{R}: u_{0}(x)>0\right\}$ is connected, then

$$
\zeta(T) \geqq \zeta(0)+\sigma_{0} T \quad \text { for all } T>0 .
$$

Proof. Fix $T>0$. By Proposition 1 and Lemma 8, there exists a $\xi_{1} \in C([0, T])$ and a $\delta>0$ such that $u\left(\xi_{1}(t), t\right) \geqq \delta>0$ for all $t \in[0, T]$. Let

$$
x_{1}=\inf \left\{\xi_{1}(t): 0 \leqq t \leqq T\right\}
$$

Choose $x_{2} \in\left(\xi_{1}(0), \zeta(0)\right)$ and $\sigma \in\left(-\infty, \sigma_{0}\right)$. Since $P_{0}$ is connected, we can find a $\mu \in(0, \delta)$ such that $u(x, 0) \geqq \mu>0$ for all $x \in\left[\xi_{1}(0), x_{2}\right]$. Moreover, since $\sigma<\sigma_{0} \leqq \sigma_{\mu}$, arguing as in the proof of Lemma 7 we can choose a real value $\beta$ such that

$$
\int_{0}^{\mu} a^{\prime}(s) /\{\sigma s+b(s)+\beta\} d s \geqq[\sigma T]_{+}+x_{2}-x_{1} .
$$

Consider the function $z(x, t)$ defined by

$$
\int_{0}^{z(x, t)} a^{\prime}(s) /\{\sigma s+b(s)+\beta\} d s=\sigma t-x+x_{2}
$$

in the closure of the domain

$$
\Omega=\left\{(x, t): \xi_{1}(t)<x<\xi_{2}(t), 0<t \leqq T\right\},
$$

where

$$
\xi_{2}(t)=x_{2}+\sigma t
$$

This function is easily verified to be a classical travelling-wave solution of equation (1.1) in $\Omega$. Furthermore, by (5.7), $u\left(\xi_{1}(t), t\right) \geqq \mu \geqq z\left(\xi_{1}(t), t\right)$ for all $t \in$ $[0, T]$, and $u(x, 0) \geqq \mu \geqq z(x, 0)$ for all $x \in\left[\xi_{1}(0), \xi_{2}(0)\right]$. In addition, $u\left(\xi_{2}(t), t\right)$ $\geqq 0=z\left(\xi_{2}(t), t\right)$ for all $t \in[0, T]$. Thus, by Lemma $4, u(x, t) \geqq z(x, t)$ for all $(x, t) \in \bar{Q}$. Whence, in particular, $\zeta(T) \geqq \xi_{2}(T)=x_{2}+\sigma T$. However since $x_{2} \in\left(\xi_{1}(0), \zeta(0)\right)$ and $\sigma \in\left(-\infty, \sigma_{0}\right)$ were arbitrary, this confirms (5.6).

Proof of Theorem 2. Since the theorem is trivially true if $\sigma_{0}=-\infty$, we may suppose without loss of generality that $\sigma_{0}>-\infty$. We may also suppose that $t_{1}=0$. Let $\left\{u_{0, k}\right\}_{k=1}^{\infty}$ be a sequence of $C(\mathbb{R})$ functions such that: (i) $u_{0, k}(x) \leqq u_{0}(x)$ for all $x \in \mathbb{R}$, (ii) $P_{0, k}=\left\{x \in \mathbb{R}: u_{0, k}(x)>0\right\}$ is a connected interval $\left(\mu_{k}, v_{k}\right)$, and (iii) $v_{k} \uparrow \zeta(0)$ as $k \uparrow \infty$. For each $k \geqq 1$, let $u_{k}(x, t)$ denote the solution of problem (1.2), (1.3) with initial data $u_{0, k}$. Set $\zeta_{k}(t)=\sup \left\{x \in \mathbb{R}: u_{k}(x, t)>0\right\}$ for all $t \geqq 0$. By Lemma 3, $u(x, t) \geqq u_{k}(x, t)$ for all $(x, t) \in \bar{H}$, and therefore $\zeta\left(t_{2}\right) \geqq \zeta_{k}\left(t_{2}\right)$. However, since $P_{0, k}$ is connected, Lemma 9 implies that $\zeta_{k}\left(t_{2}\right)$ $\geqq \zeta_{k}(0)+\sigma_{0} t_{2}$. Thus $\zeta\left(t_{2}\right) \geqq \zeta_{k}(0)+\sigma_{0} t_{2}=v_{k}+\sigma_{0} t_{2}$ for all $k \geqq 1$. Letting $k \uparrow \infty$ yields (5.1). 


\section{Continuity}

Theorem 3. The function $\zeta(t)$ is lower semicontinuous and continuous from the right on $[0, \infty)$. Moreover, if $\sigma_{0}>-\infty$ then $\zeta(t)$ is continuous on $[0, \infty)$.

Proof. The lower semicontinuity of $\zeta$ is an immediate consequence of the continuity of $u$. As to the continuity of $\zeta$ from the right, take an arbitrary $t_{0} \in[0, \infty)$. By Lemma 6,

$$
\zeta(t) \leqq \zeta\left(t_{0}\right)+\sigma\left(t-t_{0}\right)+\int_{0}^{M} a^{\prime}(s) /\{\sigma s+b(s)\} d s
$$

for all $\sigma>\sigma_{M}$ and $t>t_{0}$. Hence,

$$
\underset{t \psi t_{0}}{\limsup } \zeta(t) \leqq \zeta\left(t_{0}\right)+\int_{0}^{M} a^{\prime}(s) /\{\sigma s+b(s)\} d s
$$

for all $\sigma>\sigma_{M}$. So, letting $\sigma \uparrow \infty$,

$$
\limsup _{t \downarrow t_{0}} \zeta(t) \leqq \zeta\left(t_{0}\right) \quad \text { for all } t_{0} \in[0, \infty) \text {. }
$$

The last assertion of the theorem follows from Theorem 2.

Theorem 3 states that the interface $\zeta(t)$ is continuous when $\sigma_{0}>-\infty$. However, viewing the half-space $H$ as a plane with the $x$-axis horizontal and the positive $t$-axis pointing vertically upwards, if $\sigma_{0}=-\infty$ we cannot exclude the possibility that the interface makes sudden jumps from right to left. As an example of an equation admitting the interface $\zeta(t)$ and with $\sigma_{0}=-\infty$ we may take (1.7) with $m>n, 0<n<1$, and $\lambda>0$.

\section{Interfacial Equation}

Considering the physical situations in which equation (1.1) arises leads us to expect that the interface satisfies the identity

$$
\zeta^{\prime}(t)=-\left\{\left[(a(u))_{x}+b(u)\right] / u\right\}(\zeta(t)-, t)
$$

for all $t>0$. The next theorem indicates that in a certain sense this is indeed so.

For $t>0$, let

$$
P(t)=\{x \in \mathbb{R}: u(x, t)>0\}
$$

and set

$$
v(x, t)=-\left\{\left[(a(u))_{x}+b(u)\right] / u\right\}(x, t)
$$

for $x \in P(t)$. Define

$$
V^{-}(t)=\liminf _{\substack{x \in \zeta(t) \\ x \in P(t)}} v(x, t) \quad \text { and } \quad V^{+}(t)=\limsup _{\substack{x \uparrow \zeta(t) \\ x \in P(t)}} v(x, t) .
$$


Theorem 4. (a) If $a, b \in C^{3}(0, \delta), a^{\prime \prime \prime}$ and $b^{\prime \prime \prime}$ are locally Hölder continuous on $(0, \delta)$. For some $\delta>0$, and if $u_{0}(x)$ decreases monotonically in a left neighbourhood of $x=\zeta(0)$, then for any $t_{1} \in[0, \infty)$ and $t_{2} \in\left(t_{1}, \infty\right)$,

$$
\int_{t_{1}}^{t_{2}} V^{-}(t) d t \leqq \zeta\left(t_{2}\right)-\zeta\left(t_{1}\right) \leqq \int_{t_{1}}^{t_{2}} V^{+}(t) d t .
$$

(b) Given any $t_{1} \in(0, \infty)$ and $\varepsilon>0$ there is a $t_{2} \in\left(t_{1}, \infty\right)$ such that

$$
\left\{V^{-}\left(t_{1}\right)-\varepsilon\right\}\left(t-t_{1}\right) \leqq \zeta(t)-\zeta\left(t_{1}\right) \leqq\left\{V^{+}\left(t_{1}\right)+\varepsilon\right\}\left(t-t_{1}\right)
$$

for all $t \in\left(t_{1}, t_{2}\right]$.

Proof. Part (a) was proved in [14] with the role of (1.1) played by equation (1.7) with $m>1$ and $n \geqq(m+1) / 2$. Since this proof may be extended to the present situation with only minor modifications we shall omit it and concentrate on the proof of part (b). We use the argument that KNERR [21] used to prove the corresponding result for the porous media equation (1.8).

First, consider the left-hand inequality in (7.2). Let $x_{1}=\zeta\left(t_{1}\right)$. Set $\sigma=$ $V^{-}\left(t_{1}\right)-\varepsilon$. By Theorem 2, without loss of generality, we may suppose that $\sigma>\sigma_{0}$. Choose $\mu>0$ so small that $\sigma \in S_{\mu}$. Such a choice is justified by the remark following the statement of Theorem 1 . Next, choose $\delta>0$ so small that $u\left(x, t_{1}\right)<\mu$ for all $x \in\left[x_{1}-\delta, x_{1}\right)$, and in view of the definition of $V^{-}\left(t_{1}\right)$ so small that $v\left(x, t_{1}\right)>\sigma$ for all $x \in\left[x_{1}-\delta, x_{1}\right) \cap P\left(t_{1}\right)$. Note that by this choice of $\delta$

$$
(a(u))_{x}\left(x, t_{1}\right)<-\{\sigma u+b(u)\}\left(x, t_{1}\right)<0
$$

for all $x \in\left[x_{1}-\delta, x_{1}\right) \cap P\left(t_{1}\right)$. Thus

$$
\int_{0}^{u\left(x, t_{1}\right)} a^{\prime}(s) /\{\sigma s+b(s)\} d s>x_{1}-x
$$

for all $x \in\left[x_{1}-\delta, x_{1}\right)$. Consider the generalized (travelling wave) subsolution of equation (1.1), $z(x, t)$, defined by

$$
\int_{0}^{z(x, t)} a^{\prime}(s) /\{\sigma s+b(s)\} d s=\left[\sigma\left(t-t_{1}\right)-x+x_{1}\right]_{+} .
$$

Comparing (7.3) and (7.4), we see that $u\left(x, t_{1}\right)>z\left(x, t_{1}\right)$ for all $x \in\left[x_{1}-\delta, x_{1}\right)$. Consequently, one can choose a $t_{2}>t_{1}$ such that $\mu>u\left(x_{1}-\delta, t\right)>z\left(x_{1}-\delta, t\right)$ for all $t \in\left[t_{1}, t_{2}\right]$. However, this means that $u\left(x, t_{1}\right) \geqq z\left(x, t_{1}\right)$ for all $x \geqq x_{1}-\delta$, and $u\left(x_{1}-\delta, t\right)>z\left(x_{1}-\delta, t\right)$ for all $t \in\left[t_{1}, t_{2}\right]$. Thus, again applying Lemma 3 , $u(x, t) \geqq z(x, t)$ for all $(x, t) \in\left[x_{1}-\delta, \infty\right) \times\left[t_{1}, t_{2}\right]$. Hence we derive $\zeta(t) \geqq$ $x_{1}+\sigma\left(t-t_{1}\right)$ for all $t \in\left[t_{1}, t_{2}\right]$. This completes the proof of the left-hand inequality in (7.2).

The right-hand inequality in (7.2) may be verified analogously if we note the following.

Lemma 10. $V^{+}\left(t_{1}\right)+\varepsilon>\sigma_{0}$. 
Proof. Let $\left\{s_{i}\right\}_{i=1}^{\infty}$ denote a decreasing sequence of values in $\left(0, \sup \left\{u\left(x, t_{1}\right)\right.\right.$ : $x \in \mathbb{R}\})$ such that $s_{i} \downarrow 0$ and $-b\left(s_{i}\right) / s_{i} \rightarrow \sigma_{0}$ as $i \uparrow \infty$. Let $x_{i}=\sup \{x \in \mathbb{R}$ : $\left.u\left(x, t_{1}\right) \geqq s_{i}\right\}$. Then

$$
\begin{gathered}
v\left(x_{i}, t_{1}\right)=-\left[(a(u))_{x}\left(x_{i}, t_{1}\right)+b\left(s_{i}\right)\right] / s_{i} \\
\geqq-b\left(s_{i}\right) / s_{i} .
\end{gathered}
$$

Thus

$$
V^{+}\left(t_{1}\right) \geqq \limsup _{i \uparrow \infty} v\left(x_{i}, t_{1}\right) \geqq \sigma_{0} .
$$

Remark. If $v(x, 0)$ is defined in the sense of distributions, the conclusion of part (b) of Theorem 4 extends to $t_{1} \in[0, \infty)$.

\section{Hölder and Lipschitz Continuity Estimates}

In this last section we improve the results about continuity in the preceding sections.

Theorem 5. Suppose that (1.5) holds. Then there is a constant $C>0$ which depends only on $M$ such that for any $t_{1} \in[0, \infty)$ and $t_{2} \in\left(t_{1}, \infty\right)$,

$$
\zeta\left(t_{2}\right) \leqq \zeta\left(t_{1}\right)+\sigma_{M}\left(t_{2}-t_{1}\right)+C\left(t_{2}-t_{1}\right)^{1 / 2} .
$$

Proof. For $\sigma>\sigma_{M}$, let

$$
I(\sigma)=\int_{0}^{M} a^{\prime}(s) s /\{\sigma s+b(s)\}^{2} d s .
$$

Since by definition $\sigma_{M} s+b(s) \geqq 0$ for all $s \in(0, M]$, in the light of the remark following the statement of Theorem 1 it follows that

$$
I(\sigma) \leqq\left(\sigma-\sigma_{M}\right)^{-1} \int_{0}^{M} a^{\prime}(s) /\{\sigma s+b(s)\} d s<\infty .
$$

Moreover, by the Cauchy-Schwarz inequality,

$$
\int_{0}^{M} a^{\prime}(s) /\{\sigma s+b(s)\} d s \leqq C_{0}\{I(\sigma)\}^{1 / 2},
$$

where

$$
C_{0}=\left\{\int_{0}^{M} a^{\prime}(s) / s d s\right\}^{1 / 2} .
$$

Plainly, $I(\sigma)$ decreases monotonically as a function of $\sigma>\sigma_{M}$, and

$$
I(\sigma) \downarrow 0 \quad \text { as } \sigma \uparrow \infty .
$$


Hence

$$
I^{*}=\lim _{\sigma \downarrow \sigma_{M}} I(\sigma)
$$

is defined and positive.

Fix $t_{1}$ and $t_{2}$, and recall from Lemma 6 that for any $\sigma>\sigma_{M}$ :

$$
\zeta\left(t_{2}\right) \leqq \zeta\left(t_{1}\right)+\sigma\left(t_{2}-t_{1}\right)+\int_{0}^{M} a^{\prime}(s) /\{\sigma s+b(s)\} d s .
$$

Suppose that $t_{2}<t_{1}+I^{*}$. Then, by (8.4) and (8.5), there is a $\sigma>\sigma_{M}$ such that $t_{2}-t_{1}=I(\sigma)$. Substituting in (8.6), and using (8.2) and (8.3) in succession, we deduce

$$
\begin{aligned}
\zeta\left(t_{2}\right) & \leqq \zeta\left(t_{1}\right)+\sigma_{M} I(\sigma)+\left(\sigma-\sigma_{M}\right) I(\sigma)+\int_{0}^{M} a^{\prime}(s) /\{\sigma s+b(s)\} d s \\
& \leqq \zeta\left(t_{1}\right)+\sigma_{M} I(\sigma)+2 \int_{0}^{M} a^{\prime}(s) /\{\sigma s+b(s)\} d s \\
& \leqq \zeta\left(t_{1}\right)+\sigma_{M} I(\sigma)+2 C_{0}\{I(\sigma)\}^{1 / 2} \\
& =\zeta\left(t_{1}\right)+\sigma_{M}\left(t_{2}-t_{1}\right)+2 C_{0}\left(t_{2}-t_{1}\right)^{1 / 2}
\end{aligned}
$$

It follows that if $I^{*}=\infty$, then the theorem is proved. On the other hand, if $I^{*}<\infty$, then by (8.3) and the Monotone Convergence Theorem,

$$
\int_{0}^{M} a^{\prime}(s) /\left\{\sigma_{M} s+b(s)\right\} d s \leqq C_{0}\left\{I^{*}\right\}^{1 / 2}<\infty
$$

Hence we can let $\sigma \downarrow \sigma_{M}$ in (8.6) to deduce

$$
\zeta\left(t_{2}\right) \leqq \zeta\left(t_{1}\right)+\sigma_{M}\left(t_{2}-t_{1}\right)+C_{0}\left\{I^{*}\right\}^{1 / 2} .
$$

But then by using (8.7) for $t_{2}<t_{1}+I^{*}$ and (8.8) for $t_{2} \geqq t_{1}+I^{*}$, we prove the theorem in this case also. The constant $C=2 C_{0}$.

Remark. The coefficient $\sigma_{M}$ in (8.1) is the best possible.

This remark can be verified by considering the explicit generalized solution

$$
\int_{0}^{u(x, t)} a^{\prime}(s) /\left\{\sigma_{M} s+b(s)\right\} d s=\left[\sigma_{M} t-x\right]_{+},
$$

when $\sigma_{M}>\sigma_{M^{\prime}}$ for all $M^{\prime}>M$.

Theorem 6. (i) Suppose that (1.5) holds. Then given any $\tau>0$, there is a constant $C>0$, which depends only on $M$ and $\tau$, such that for any $t_{1} \in[\tau, \infty)$ and $t_{2} \in$ $\left(t_{1}, \infty\right)$

$$
\zeta\left(t_{2}\right) \leqq \zeta\left(t_{1}\right)+C\left(t_{2}-t_{1}\right) .
$$

(ii) Suppose that there is a constant $L \geqq \sigma_{M}$ such that (2.9) holds for all $x_{1}, x_{2} \in \mathbb{R}$ with $x_{2}>x_{1}$, then there is a constant $C>0$ which depends only on $M$ and $L$ such that (8.9) holds for any $t_{1} \in[0, \infty)$ and $t_{2} \in\left(t_{1}, \infty\right)$. 
This theorem follows directly from the upper bounds in Theorem 4 (ii) and Proposition 2 if one observes that under the conditions for the existence of the interface $\zeta(t)$, necessarily $[-b(s) / s]_{+} \in L^{\infty}(0, M)$.

\section{References}

1. S. Angenent, Analyticity of the interface of the porous media equation after the waiting time, Mathematical Institute University of Leiden Report 30 (1985), $12 \mathrm{pp}$.

2. S. N. ANTONCEv, On the localization of solutions of nonlinear degenerate elliptic and parabolic equations, Soviet Math. Dokl. 24 (1981), 420-424. Translation of: Dokl. Akad. Nauk SSSR 260 (1981), 1289-1293.

3. D. G. Aronson, L. A. Caffarelli \& J. L. Vazquez, Interfaces with a corner point in one-dimensional porous medium flow, Comm. Pure Appl. Math. 38 (1985), 375404.

4. D. G. Aronson \& J. L. VÁzQuez, Eventual $C^{\infty}$-regularity and concavity for flow in one-dimensional porous media, University of Minnesota Institute for Mathematics and its Applications, Preprint 290 (1987), 28 pp.

5. J. Bear, Dynamics of Fluids in Porous Media, American Elsevier Publishing Company, New York London Amsterdam, 1972.

6. P. Benilan \& J. L. VAzQuez, Concavity of solutions of the porous medium equation, Trans. Amer. Math. Soc. 299 (1987), 81-93.

7. J. BUCKMASTER, Viscous sheets advancing over dry beds, J. Fluid Mech. 81 (1977), $735-756$.

8. L. A. CAfFarelli \& A. Friedman, Regularity of the free boundary for the one-dimensional flow of gas in a porous medium, Amer. J. Math. 101 (1979), 1193-1218.

9. S. ChANDRASEKHAR, Stochastic problems in physics and astronomy, Rev. Modern Phys. 15 (1943), 1-89.

10. J. I. Diaz \& R. KerSner, Non existence d'une des frontières libres dans une équation dégénérée en théorie de la filtration, C. R. Acad. Sci. Paris Sér. I Math. 296 (1983), 505-508.

11. J. I. DiAz \& L. Véron, Compacité du support des solutions d'équations quasi linéaires elliptiques ou paraboliques, C. R. Acad. Sci. Paris Sér. I Math. 297 (1983), 149-152.

12. J. I. DiAZ \& L. Veron, Local vanishing properties of solutions of elliptic and parabolic quasilinear equations, Trans. Amer. Math. Soc. 290 (1985), 787-814.

13. L. C. EVANS \& B. F. KNERR, Instantaneous shrinking of the support of nonnegative solutions to certain nonlinear parabolic equations and variational inequalities, Illinois J. Math. 23 (1979), 153-166.

14. B. H. GILDING, Properties of solutions of an equation in the theory of infiltration, Arch. Rational Mech. Anal. 65 (1977), 203-225.

15. B. H. Gilding, A nonlinear degenerate parabolic equation, Ann. Scuola Norm. Sup. Pisa Cl. Sci. (4) 4 (1977), 393-432.

16. B. H. GILDING, Improved theory for a nonlinear degenerate parabolic equation, submitted for publication. Also appearing as: Twente University of Technology Department of Applied Mathematics Memorandum 587 (1986), 42 pp.

17. K. Höllig \& H.-O. KreIss, $C^{\infty}$-regularity for the porous medium equation, Math. $Z$. 192 (1986), 217-224.

18. A. S. Kalashnikov, The occurrence of singularities in solutions of the non-steady seepage equation, U.S.S.R. Comput. Math. and Math. Phys. 7 (1967), 269-275. Translation of: Zh. Vychisl. Mat. i Mat. Fiz. 7 (1967), 440-444. 
19. A. S. Kalashnikov, The nature of the propagation of perturbations in processes that can be described by quasilinear degenerate parabolic equations (in Russian), Trudy Sem. Petrovsk. 1 (1975), 135-144.

20. R. KeRSNER, Filtration with absorption: necessary and sufficient condition for the propagation of perturbations to have finite velocity, J. Math. Anal. Appl. 90 (1982), $463-479$.

21. B. F. KNERr, The porous medium equation in one dimension, Trans. Amer. Math. Soc. 234 (1977), 381-415.

22. O. A. Oleinik, A. S. Kalashnikov \& Chzhou Y.-L., The Cauchy problem and boundary problems for equations of the type of non-stationary filtration (in Russian), Izv. Akad. Nauk SSSR Ser. Mat. 22 (1958), 667-704.

23. L. A. Peletier, A necessary and sufficient condition for the existence of an interface in flows through porous media, Arch. Rational Mech. Anal. 56 (1974), 183-190.

24. L. A. Peletier, On the existence of an interface in nonlinear diffusion processes, Ordinary and Partial Differential Equations (edited by B. D. SLEEMAN \& I. M. Michael), Lecture Notes in Mathematics 415, Springer-Verlag, Berlin Heidelberg New York, 1974, pp. 412-416.

25. M. H. Protter \& H. F. Weinberger, Maximum Principles in Differential Equations, Prentice-Hall, Englewood Cliffs, New Jersey, 1967.

26. J. L. VÁZQUEZ, The interfaces of one-dimensional flows in porous media, Trans. Amer. Math. Soc. 285 (1984), 717-737.

27. J. L. VÁzQUEZ, $C^{\infty}$-regularity of solutions and interfaces of the porous medium equation, in preparation.

Faculty of Applied Mathematics

University of Twente

Enschede

The Netherlands

(Received April 10, 1987) 